\title{
The Comparative Change of Self-Efficacy in Nursing Students After Verbal De-Escalation Education
}

\author{
Tina Hostetler ${ }^{1}$ \\ ${ }^{1}$ Memorial Epworth (Psychiatric) Educational Services, Beacon Health System, South Bend, Indiana, USA \\ Correspondence: Samuel P. Abraham, Associate Professor of Nursing, Bethel University, 1001 Bethel Circle, \\ Mishawaka, Indiana, USA.
}

Received: March 9, 2020

Accepted: March 26, 2020

Online Published: March 30, 2020

doi:10.20849/ijsn.v5i1.730

URL: https://doi.org/10.20849/ijsn.v5i1.730

\begin{abstract}
Background: The incidence of workplace violence has continued to occupy a significant place in healthcare at a rate nearly double that in other industries. Those providing direct bedside care are among the most vulnerable to violence, including nursing students. Many students report experiencing verbal or physical aggression prior to graduating from nursing school. Purpose: The purpose of this quantitative prospective experimental study was to measure the level of self-efficacy of nursing students' verbal de-escalation skills before and after verbal de-escalation training, to see if the training made a measurable effect on the level of self-efficacy. Method: Seventeen nursing students in their psychiatric-mental health course rotation completed a pre- and post-intervention survey based on Thackrey's Confidence in Coping with Patient Aggression scale (1987) to measure self-efficacy. The intervention included up to 90 minutes of interactive verbal de-escalation training. Result: A statistically significant increase in self-efficacy scores was noted from pre-intervention $(M=39, S D=$ 13.5) to post-intervention $(M=60.9, S D=14.3), t(16)=6.92, p<.001$ (two-tailed). The mean increase in CCCPA scores was 21.8 with a $95 \%$ confidence interval ranging from 28.6 to 15.2 . The eta squared statistic (0.46) indicated a large effect size. Conclusion: Verbal de-escalation training in all nursing programs is recommended.
\end{abstract}

Keywords: verbal de-escalation, workplace violence, nursing student

\section{Introduction}

This research focused on the effect of verbal de-escalation training of nursing students during a psychiatric clinical rotation, as measured by the students' level of self-efficacy before training compared with afterward. The study outlines the extent of workplace violence issues in the United States and around the world, and the historical context of healthcare violence, with a brief review of research in the student nurse population. An introduction to Bandura's Self-Efficacy model (1977) as a theoretical framework is included.

This section of research also provides an in-depth explanation of study details, including the research project design, sample selection, and generation of data using Thackrey's Confidence in Coping with Patient Aggression tool (1987). Lastly, major findings of the research, implications for nursing practice related to verbal de-escalation training, and suggested directions for further research into student nurse de-escalation training and workplace violence prevention are presented.

\subsection{Instrumentation}

A variety of instruments were used within the reviewed research, both to evaluate the educational module delivered, and the learning effect upon the nursing student participants. Kraiger's Learning Model (Kraiger, Ford, \& Salas, 1993) was used by Beech and Leather (2003), and then Beech individually (2008), claiming that model to be the gold standard for others to meet. The authors of the model stressed that learning outcomes were multidimensional, and the best way to evaluate outcomes for attainment was by considering changes made in cognitive or affective realms, or in skill abilities (Kraiger, Ford, \& Salas, 1993).

More than one study utilized an instrument of their own making, including the De-Escalating Aggression Behavioral Scale (Nau, Needham, Dassen, \& Halfens, 2009), and Perception of Aggression Scale, Short Form (Needham, Abderhalden, Dassen, Haug, \& Fischer, 2004). The ability of student nurses to calm an agitated patient was the intended item to be measured by the De-Escalating Aggression Behavioral Scale. The Perception 
of Aggression Scale was formulated to measure nurses' attitudes toward aggressive patients. As noted previously, the Confidence in Coping with Patient Aggression scale measured the level of efficacy toward dealing with an agitated patient. Each of these tools could be an appropriate choice, depending on the characteristic or behavior requiring further exploration.

\subsection{Gaps in the Research}

Lack of a control group was often noted in the literature reviewed (Beech, 1999; Beech \& Leather, 2003; Brann \& Hartley, 2017; Jonas-Dwyer et al., 2017; Nau, Dassen, Needham, \& Halfens, 2009). The wisdom or ethical consideration of giving no education on de-escalation techniques to control participants, which may prevent injury, was not addressed (Gurkan \& Komurcu, 2017). A minimum amount of information could have been relayed which would not have interfered with study results to the control groups, such as mandating the immediate departure from the area of any student within 15 feet of an agitated or aggressive patient.

\subsection{Gaps in Instrumentation}

Violence in health care is on the rise, and nursing students are often viewed as more vulnerable to possible harm than other care providers (Beech, 1999; Magnavita \& Heponiemi, 2011). Whitley, Jacobson, and Gawrys (1996) suggest that the safety of student nurses is as important as the patients that are served and cannot be sacrificed. Minimal research has been conducted over the last 40 years, and even fewer efforts have been directed toward the safety of nursing students. The small amount of research to date has been uncoordinated and noncumulative, leading to a piecemeal effect of outcomes. Gaps in method and instrumentation have added fuel to the issue, and it is no wonder that inconsistencies exist in the inclusion, content, or placement of de-escalation and anti-violence measures in nursing curriculum (Brann \& Hartley, 2017; Hopkins, Fetherston, \& Morrison, 2014).

\subsection{Statement of the Problem}

Nursing students attending clinical rotation on inpatient psychiatric units may not have training in techniques to identify and protect themselves in the presence of an agitated or aggressive patient. The student's necessary task of quickly assessing the patient and establishing therapeutic rapport exposes the student to a breadth of psychiatric symptoms which may include paranoia, psychosis, hallucinations, and homicidal thoughts, often fueled by substance use (Nau, Dassen, and Halfens, 2009). The student may lose sight of the therapeutic environment in the drive to complete course tasks, missing essential queues from the patient that the interaction needs to go in a different direction, take a break, or end.

The nursing student leaving an agitated patient on the unit and walking away will not facilitate learning therapeutic communication skills for later clinical or post-licensure experiences. The inability to recognize or stop impending violent action leads three in ten nurses to physical abuse, according to one study (Speroni, Fitch, Dawson, Dugan, \& Atherton, 2014). For more than 40 years the literature has mentioned violence against nurses as if it were a fad. Failing to teach proper action, before violence and agitation, promotes acceptance and continuation of the current levels of viciousness against nurses and all healthcare workers worldwide. Teaching nursing students aggression management techniques provides a tool for successful verbal de-escalation, useful in both the mental health and medical healthcare environments, for the rest of a nurses' career (Nau et al., 2010).

\subsection{Purpose of the Project}

The purpose of this quantitative prospective experimental study was to measure the level of self-efficacy of nursing students' verbal de-escalation skills before and after verbal de-escalation training, to see if the training made a measurable effect on the level of self-efficacy. Bandura (1977) theorized that the greater the level of self-efficacy, the greater the amount of behavioral functioning, such as that required for verbal de-escalation.

The decrease in verbal and physical abuse of nurses will require a shift in organizational culture. One of the best ways to begin this culture shift is through education. Verbal de-escalation skills education assists in early recognition of patient agitation while learning techniques to verbally calm the patient. Knowledge of the opportune time to signal for help from surrounding staff, and identification of the proper time to exit the unit and allow more experienced staff to intervene, are ideal outcomes (Nau, Dassen, Needham, \& Halfens, 2009).

As the population of interest, nursing students often lack life experience, including appropriate interventions to correctly calm an agitated patient, or promptly remove themselves from the volatile situation (Truman et al., 2013). Five nursing schools attended the clinical rotation site. The primary variables of focus were the de-escalation training (independent variable), and the level of self-efficacy (dependent variable). The research question was clear: For nursing students in their psychiatric clinical rotation, was there a change in the level of self-efficacy in verbal de-escalation skills before verbal de-escalation training compared to immediately afterward. The PICOT-formatted question was as follows: 
- $\quad$ P: In nursing students with less than 6 months of employment-related clinical experience,

- I: did verbal de-escalation education training

- C: affect confidence scores after training compared to prior

- O: resulting in a change in de-escalation self-efficacy while coping with patient aggression

- $\quad \mathrm{T}$ : during the psychiatric clinical rotation?

\subsection{Significance of the Project}

Nursing students' knowledge of how and when to use verbal de-escalation skills is an important tool in the nurses' list of intervention choices (Cowin, Davies, Estall, Fitzgerald, \& Hoot, 2003). This skill is being lost as older mental health nurses retire and new nurses arrive on the units without this crucial information (Cowin et al., 2003). Without experience or education in how to interact with patients exhibiting mental illness, the knowledge of which situations to avoid, when to proceed, when to slow down, what actions or movements have meaning, and verbal and nonverbal cues, may be missed or misinterpreted (Cowin et al., 2003).

Many patients have a complex mix of overlapping layers, and with just under $20 \%$ of the populace living with a mental illness, one of the layers may be psychiatric in nature (Centers for Disease Control and Prevention, 2014; Unsworth, McKeever, \& Kelleher, 2012). Early assessment of agitation, and early intervention utilizing de-escalation skills will assist the patient to feel supported, knowing the student nurse will help the patient to maintain control of themselves (Cowin et al., 2003; Lavell, Stewart, James, Richardson, Renwick, Brennan, \& Bowers, 2016). This therapeutic process can have an immediate effect of putting the patient at ease, and therefore useful for patients, with a calming effect on medical conditions.

On a collegiate level, nursing schools may be reluctant to utilize the psychiatric inpatient units, due to the students' lack of communication skills and knowledge in caring for agitated patients. This uncertainty has caused some schools to either limit or avoid using inpatient psychiatric units as clinical sites to decrease the risk of student injury (Quail, Bundage, Spitalnick, Allen, \& Beilby, 2016), which could be remedied through verbal de-escalation training. A student who had a positive psychiatric clinical rotation is more likely to gain meaningful experience for enhanced learning (Cleary, Horsfall, \& DeCarlo, 2006).

The healthcare organization with workplace violence awareness and procedures in place to lessen aggression and agitation will realize a decrease in the severity of aggressive events (Martinez, 2016). One study noted $85 \%$ of rapid response calls for agitation were resolved successfully via verbal de-escalation and other noncoercive medication measures (Martinez, 2016). Nurses feel more confident with patient interactions after verbal de-escalation training and are more comfortable working around potentially violent patients (Lavelle et al., 2016; Martinez, 2016; McGowan, Wynaden, Harding, Yassine, \& Parker, 1999). A positive effect on engagement and feelings of loyalty should result as students not only see the clinical site recognizing the issue but taking proactive steps to assist in protecting them (Brann \& Hartley, 2017).

The nursing profession will benefit from the student nurse learning verbal de-escalation skills and becoming confident in their use (Lavell et al., 2016). As future nurses, the rates of injury and loss of work productivity should be less, as the patient's agitation will be recognized and treated early (Simon \& Hurvitz, 2014). When agitation is not allowed to escalate to violence, nurses may be less inclined to leave their positions prematurely, thereby having a positive impact on retention rates, a crucial factor in today's nursing shortage times (Martinez, 2016). Additionally, nurses will be less stressed and more willing to fully care for their patients (Brann \& Hartley, 2017).

In current and future society, learning methods to de-escalate an agitated patient will benefit the nurse in any environment, in any country. Prior experience with psychiatric patients as a student nurse will be beneficial in caring for this self-neglectful population (Unsworth, McKeever, \& Kelleher, 2012). The global mental health community at large will begin to benefit when medically compromised psychiatric patients are treated the same as those without significant mental issues, and not labelled as the one who is every nurse's last pick for the daily assignment.

This study contributed essential knowledge to the current research on violence toward nursing students in healthcare, by utilizing a nursing student population in a psychiatric clinical rotation, a psychometrically sound survey instrument, and an evidence-based educational program. The addition of this research has helped to address the effectiveness of verbal de-escalation training as a nursing student intervention. 


\section{Nature, Scope, and Limitations of the Project}

The research project was a comparative, prospective study focused on the change in level of self-efficacy in verbal de-escalation skills from prior to de-escalation training, to after the education, as scientifically rigorous as possible, while keeping the students' safety at the forefront of the research design and implementation.

\subsection{Nature}

\subsubsection{Research Design}

A pre- and post-test design was planned for this study. A questionnaire was administered prior to verbal de-escalation education. The same questions were. Queried to participants following the education, minus the demographics. The results of the surveys were then compared for a possible change in levels of self-efficacy. A higher level of self-efficacy was a better signal of intent to act, including the intent to put into place the principles of verbal de-escalation that have been taught to nursing students (Bandura, 1977).

\subsubsection{Data Collection}

After informed consent was obtained, the plan was for the students to be given a simple number and asked to self-select a code consisting of a meaningful four-digit number and the first two letters of the mother's maiden (last) name. The student would write this code on the paper questionnaire, which consisted of seven demographic questions and the ten questions of the Confidence in Coping with Patient Aggression scale (Thackrey, 1987).

The initial survey occurred immediately prior to the verbal de-escalation training. After the training, the questionnaire was administered again with the student entering the same meaningful code on their paper. All questionnaires were collected and placed in a locked safety box until the data analysis.

\subsubsection{Instrumentation}

Quantitative data were collected utilizing Thackrey's Confidence in Coping with Patient Aggression scale (1987). This confidence scale was a ten-item self-reporting instrument utilizing Likert-type responses on a score of 1 to 11 , with 1 being the lesser confidence side of the scale and 11 being the greater confidence side. The scale was previously tested for internal consistency, with a Cronbach's alpha score of 0.92 , considered highly reliable. This scale was not widely published in the research literature but was specific to this clinical scenario.

\subsubsection{Data Analysis}

The secured data box was opened, and the two questionnaires matched according to the student's self-selected code. Data were entered in a Microsoft Excel spreadsheet and scored using the Statistical Package for the Social Sciences, version 25. The Paired Samples t-test was planned for use to ascertain the difference in the scores from the two time periods. A level of significance of $p<0.05$ was used to test for statistical significance. A higher final level of self-efficacy is a better signal of intent to act, including the intent to put into place the principles of verbal de-escalation that had been taught to nursing students (Bandura, 1977).

\subsubsection{Data Management}

All data were secured in a locked portable safe when traveling from the schools of nursing, until the permanent locked filing cabinet location was reached. The data will be stored under double lock and key in the permanent location for a period of no less than five years. Results of the analysis were reported back to each nursing school, according to the method preferred by the institution.

\subsubsection{Scope}

The population under study for this comparative prospective project was a convenience sample of nursing students from four surrounding schools of nursing, with a potential of 60 students in the combined full and half-semesters. All the intended sample students attended clinical rotations at a local 66-bed, four-unit psychiatric hospital, at differing times throughout the week. All students rotated through at least two of the adult units, though not all the schools chose to rotate through the adolescent unit, mainly due to its physical location in a separate building across the street.

Only one school was present on the psychiatric units at a time. The questionnaires were delivered manually on paper, on location at each of the four schools of nursing, in Indiana and in Michigan. Students who did not complete both questionnaires were eliminated from the final result, as were those who do not complete the questionnaires in their entirety. Each of the ten Likert-type questions were answered in whole numbers, from one through eleven, per explicit instructions. Those participants who failed to answer the questions per instructions were also eliminated from the final tally. 
The verbal de-escalation education occurred at the schools of nursing, using the same program for each school. All students were given the same information. An allowance of three weeks was made from the time of completed data collection to final result compilation for data calculation and extrapolation, with the final data analysis available within six weeks after the initial survey.

\subsubsection{Limitations}

The primary limitation this study faced was the small convenience sample of nursing students. Though there was a potential for up to 100 students for the psychiatric clinical rotation, enrollment rates may have affected how many students were included in any given semester. For most programs, the psychiatric clinical was located in the middle or near the end of the nursing program which may have affected sample size due to attrition or programmatic course sequence issues.

The second possible limitation was the type and amount of patient exposure each of the various nursing schools allowed or scheduled in the psychiatric rotation. For instance, one school may spend one clinical day and one on-campus simulation day per week, while another nursing school may choose to spend two days a week at the clinical site, with most of that time on the acute adult (psychiatric intensive care) unit. The second school might feel the verbal de-escalation training was of greater value if there were more chances to utilize the training activities. Each of these two scenarios may affect the generalizability of the study's findings and recommendations for future research.

\subsubsection{Delimitations}

Much of the limited research in existence regarding de-escalation training programs with nursing students at least partially utilized a qualitative approach in the study (Beech, 1999; Beech, 2008; Jonas-Dwyer, et al., 2017; Brann \& Hartley, 2017; Magnavita \& Heponiemi, 2011; Nau, Dassen, Halfens, \& Needham, 2007). Polling a program's attendees immediately after an educational module regarding the utility of learned information often results in a generic positive reaction (Beech \& Leather, 2003).

Additionally, relying on the memory of aggressive events from several weeks earlier for survey answers, and asking the student to avoid sullying those memories with any other intervening opinion is a difficult task. This study did not rely on a qualitative, retrospective approach for those two reasons, and because of the lack of academic rigor associated with statistics derived from qualitative research into this area.

The study did not test knowledge acquisition via formal exams following the verbal de-escalation training, as test scores have proven too weak a predictor of future success in verbal de-escalation of patients (Beech \& Leather, 2003). Additionally, the proficiency of students in similar areas, such as therapeutic communication and relationship-building strategies, occurs throughout the clinical and in simulation settings (Fiedler, Breitenstein, \& Delaney, 2012). Assisting students to become more confident in their skills performance is also an important faculty support responsibility (Fiedler, Breitenstein, \& Delaney, 2012), and for this reason, self-efficacy of the student was measured in lieu of scoring the actual performance of the skill.

An increase in self-efficacy accompanies an increase in motivation and feelings of confidence in ability (Bandura, 1997). The number of reports of aggressive or agitated episodes were not used as an indicator of successful verbal de-escalation and a sign of high self-efficacy, as some studies have shown people become angry just as often, regardless of the skills of the staff (Heckemann et al., 2015).

Lastly, this study was not intended to assign blame for the lack of nursing student education in anti-aggression methods. Previous research had been conducted with relevant data illuminated, wherein the researchers accused the opposite party of shirking their duties regarding nursing student security. The schools of nursing and the healthcare organization worked cooperatively on this study, each one understanding their role in student, patient, and unit safety.

\subsubsection{Feasibility}

There were no costs for this research project. A major consideration in the viability of the study may be an unwillingness of the schools of nursing to allow a control group of students who would not be instructed in verbal de-escalation techniques. The control group would be receiving the usual and customary training routinely given prior to a mental health clinical rotation. If the schools believed the verbal de-escalation training would greatly improve the safety of all students, there may be a reluctance or refusal to allow variability in student education.

Finally, there was a possibility the schools would not grant researcher access to the students, or that the schools would require a lengthy Institutional Review Board process prior to granting permission to conduct research on 
nursing student participants. Another concern was the refusal of a school to allow data derived from nursing student participants to be published or made public in some other fashion. Each of these circumstances would impact the study and potentially weaken the structure or outcome of the research.

\section{Theoretical Framework}

The theoretical framework for this proposed study rests on the structural tenets of Bandura's Self-Efficacy Theory (1977).

\subsection{History and Description of Self-Efficacy Theory}

Albert Bandura developed Self-Efficacy Theory in 1977 at Stanford University while working in the field of psychology on factors influencing change in individuals (Bandura, 1977). He surmised that although mental and physical repetitive processes will both yield a change, there is an underlying cognitive mechanism which impacts the level of achievable success. Bandura's Self-Efficacy Theory postulates that "personal mastery expectations are the primary determinants of behavioral change" (Sherer, Maddux, Mercandante, Prentice-Dunn, Jacobs, \& Rogers, 1982).

The expectation of mastery or control in either mental or physical skill is influenced by specific differences in the way past occurrences were recorded as cognitive events in the brain. These cognitive events may change the amount of motivation, effort, and behavior one produces in a situation where deliberate action is required (Bandura, 1977).

\subsubsection{Self-Efficacy Components}

As memories of life scenarios are internalized mentally, they become cognitive events, or bits of persons and circumstance which stand out and are remembered anecdotally (Bandura, 1977). According to Bandura, such cognitive events are filed away in the brain in four different categories: performance accomplishment, vicarious experience, verbal persuasion, and emotional arousal. These four categories individually or collectively influence the level of mastery or self-efficacy that each person believes they possess in any situation. Each of these categories and their contents adds or subtracts from the individual belief in the self-efficacy judgment, so that the entire concept is always potentially fluid, according to personal and environmental input (Bandura, 1997).

The first of the four tenets of the self-efficacy judgment is derived from performance accomplishment or those actions achieved by an individual (Bandura, 1977). Persistently engaging in practice of a skill will lead to greater feelings of comfort with the undertaking of a learned behavioral response activity. Performance accomplishments, therefore, are an exceptionally strong influencer, and can affect self-efficacy in either a positive or negative way. Repeated successes will boost self-efficacy judgment, while frequent failures will have the opposite effect (Bandura, 1977). Failures which occur early in an attempted new process or skill are especially detrimental to self-efficacy and feelings of mastery.

Vicarious experience is the second concept, which is the observation of acts performed by others, such as the demonstration of a new skill or process. This type of cognitive event can also be produced by the acting out of a new skill, either by live or symbolic modeling of the process or behavior (Bandura, 1977). Strong influencers are the threatening or stressful scenarios acted out by others, which is why case studies of others' actions are occasionally more clearly remembered than one's own personal experiences (Bandura, 1977).

Formal lecture and informal communications are often formats for verbal persuasion, the third concept in the Self-Efficacy Theory (Bandura, 1977). Persons can be led into believing they can master a skill or process previously considered impossible. This type of influence tends to be weaker, due to not being based in first-person experience, especially if there has been a long history of previous failures (Bandura, 1977). Research has proven that persons with phobias may be helped by overexposure and desensitization, but only if they are explicitly informed throughout the process of the desired outcome (Bandura, 1977).

The fourth concept in Bandura's Theory is emotional arousal (Bandura, 1977). Situations which elicit extreme emotion, such as those which move one to tears, shaking with fear, or sweating with anxiety, have a stronger influence than the mundane. However, one caveat to this influencer is that if the level of arousal is too high, the person will become debilitated by a belief in lack of self-efficacy, (failure by inability). Fear breeds greater fear, making it possible to scare oneself out of attempting a new skill or process, or moving out of the rut in which one is stuck.

These four concepts- performance accomplishments, vicarious experience, verbal persuasion, and emotional arousal- together inform the self-efficacy judgment, either adding or subtracting to, perceived ability. Stimuli from the environment colors one's ability to self-correct the self-efficacy judgment, which then feeds into the 
likelihood to perform the appropriate behaviors correctly in a prompt manner. Bandura identified that if a learned action could be used to cope with potential threats, fear would be decreased or eliminated, and the environment deemed less stressful.

However, negative influences in any of the four tenets of the theory may also diminish the level of self-efficacy. The beliefs in one's abilities is a two-way street. This premise fits well with the goals and purpose of verbal de-escalation training, and with the psychiatric nursing student clinical rotation.

\subsubsection{Project Alignment With Self-Efficacy Theory}

Self-efficacy measurements are one of the most prominent indicators of behavioral change (Sherer et al., 1982). The decision to act is strongly influenced by one's belief in self-efficacy, and if completed successfully, a new skill or process builds positive feelings to continue. Likewise, the greater one's level of self-efficacy, the longer an appropriate responsive behavior will be sustained (Bandura, 1994). Since the project aimed to measure the difference in levels of self-efficacy in nursing students before and after verbal de-escalation training and given the fact that verbal de-escalation is a specific behavior, Bandura's Self-Efficacy model was an appropriate theoretical framework on which to base this project.

In this research project, performance accomplishment influences were achieved during verbal de-escalation training, primarily through scenarios whose premise is that of a role-play interaction on a psychiatric unit. Various potential patient situations were presented in a low-key manner, so that the nursing student may interact without feeling the stress of an actual agitated patient. Key concepts were taught and reinforced during this time. As one becomes accustomed to the stimulus activity (scenario presented), the appropriate behavioral response becomes almost automated; the level of comfort is linked to the feeling of self-efficacy (Bandura, 1997).

Vicarious experience was accomplished during verbal de-escalation training when a student or the trainer demonstrated a skill or verbal response in front of others or was asked to explain a concept to the group. Vicarious experience may also be demonstrated through the use verbal de-escalation video clips and stories of actual experiences relayed to the group. Viewed role-playing of others in difficult situations is also an effective way to influence self-efficacy through vicarious influence. The student learns from others' successes and mistakes, and internalizes information in a meaningful way (Bandura, 1977).

Teaching and explaining the key concepts of verbal de-escalation to the group facilitates the use of verbal persuasion (Bandura, 1977). Written materials reinforce the concepts as plausible working intervention tools to the students during the psychiatric clinical rotation. Being truthful about the potential for violence on the unit and the safety measures enforced by all unit staff helped to ease the fears and anxieties of the student. Nursing research demographics state most nursing students are young, though middle-aged and second-career nurses are entering schools in larger numbers (Jonas-Dwyer et al., 2017). The nursing student with few coping skills is poorly equipped to handle stressful situations, including agitated patients in the psychiatric clinical rotation. Acknowledgement of that fact, with an intent to resolve deficiencies, provides a sense of transparency in teaching verbal de-escalation.

Emotional arousal accompanies the best learning experiences, in verbal de-escalation training, when utilized in realistic group role play. A simulated patient acted out verbally and threatened physical violence, while the group attempted to contain the patient and situation, experiencing the emotions and barriers which may be present in an actual violent encounter. Fight-or-flight physiological responses are triggered, senses are heightened, and information is absorbed quickly by the student, in preparation for response. Pointing out the occurrence of debilitation due to over-arousal is also an important teaching moment and learning experience, as a self-awareness of triggering signs and symptoms may be a signal to take aversive action in some students.

Bandura's theory focuses on the perception of the person's ability (self-efficacy), and not necessarily on the actual result of an action. The greater one's level of self-efficacy, the more likely one is to act as the training has educated one to, which is to verbally calm the patient (Bandura, 1994). Self-efficacy is the result of a level of confidence and motivation in one's abilities. The beliefs one embodies regarding the strength of personal self-efficacy in some part determines the career path one chooses, as capabilities are pondered (Bandura, 1977).

Without a rudimentary mastery of coping skills and a level of self-efficacy to use them, further occupational and educational training are no more than added layers smothering the person within (Bandura, 1997). One's level of self-efficacy affects life choices and especially during stressful and potentially violent times on the inpatient psychiatric unit, these need to be the correct choices. Therefore, verbal de-escalation training may teach the skills needed to effectively handle aggressive patients, while helping to increase self-efficacy in dealing with this patient population. 


\subsection{Definition of Terms}

Abuse: Maltreatment, physical or otherwise, which is intended to harm (Abuse, 2017). Examples may include physical, emotional, or verbal abuse. Verbal abuse may be further defined as purposeful threats of violence, coercion, theft, or damage to one's reputation intended to cause damage, fear, or impaired decision-making (Whitley, Jacobsen, and Gawrys, 1996).

Aggression: Behavior that is meant to cause injury to another person, whether of a physical or emotional nature. Aggression may also be direct or indirect, such as the destruction of another's property (Beech \& Leather, 2003).

Clinical rotation: The time during which a student practices in an instructor-supervised environment on real patients. The clinical rotation may take place in many settings, such as the acute care hospital, an extended-care facility, or a community setting (Fiedler, Breitenstein, \& Delaney, 2012).

Confidence: A feeling of being certain, such as a feeling of confidence in successful ventures (Confidence, 2017).

Emotional abuse: The effect of intentionally negative actions or language which causes feelings of incompetence, helplessness, and worthlessness (Brann \& Hartley, 2017).

Self-efficacy: The future-oriented belief in one's ability to achieve, whether it be process, procedure, or behavioral intervention such as de-escalation. Bandura (1977) states self-efficacy is utilizing knowledge and skill appropriately in stressful situations. Roadblocks are not viewed as obstacles to stop progress, but as mere impediments (Bandura, 1997). A feeling of confidence combined with the knowledge of skill attainment.

Therapeutic milieu: Any space which is optimal for healing, especially for the mental health patient. Positive talk, hope, safety, and patient-centered care are a few aspects of therapeutic milieu care (Mahoney, Palyo, Napier, \& Gordano, 2009).

Verbal de-escalation: Used as a first-line defense during escalating aggression, verbal de-escalation is the use of verbal communication and body language to calm a patient's behavior (Lavelle et al., 2016).

Workplace violence: Acts which are directed toward persons during employment duties or traveling to or from work, including physical assault and verbal threats of violence (Brann \& Hartley, 2017).

\subsection{Summary}

Workplace violence in the healthcare field has continued to rise annually at alarming rates (Centers for Disease Control and Prevention, 2015). Nurses are targeted more than any other discipline (Campbell, Messing, Kub, Agnew, Fitzgerald, Fowler, and Bolyard, 2011) and by extension, nursing students are also at high risk (Nau, Dassen, Halfens, \& Needham, 2007). Lack of worldly knowledge combined with a culture of blind ignorance from hospitals and nursing schools regarding the realities of violence, causes a knowledge gap which will continue to endanger our newest and brightest future nurses. Working in the psychiatric unit increases the risk of violence due to the nature of the patient being treated (Campbell et al., 2011), and mental health clinical rotations face the same chance of violence.

Nursing students are expected to efficiently perform assessments, initiate therapeutic communication, and handle incidents of patient agitation or aggression without regard to lasting effects on the student or the patient. Many nursing students are young and often inexperienced in dealing with aggression, lacking appropriate assessment and interpretation of situational queues (Nau, Dassen, Needham, \& Halfens, 2009), coping skills, or self-protection measures (Needham et al., 2005).

De-escalation is the first-line treatment for a patient who becomes aggressive and threatening and is preferable over seclusion or physical restraint (Lavelle et al., 2016). The goal of this type of training is safety for the staff, patient, and others in the immediate area. The core concepts of de-escalation need to be remembered on a long-term basis. Previous research follow-up was 18 months at the most, but principles of the education should be carried on into the nurses' career (Brann \& Hartley, 2017). Relatively little research has been conducted with nursing students learning verbal de-escalation skills, and only one study has been found which occurred in the United States (Brann \& Hartley, 2017), laying the foundation for the proposed study.

Measuring levels of self-efficacy before and after verbal de-escalation training will add to the body of nursing knowledge by providing specific details of training content, cultural context, and clues into the cognitive processes of nursing students in the United States. This information could be used to develop training materials for nursing students and staff in other healthcare environments, such as outpatient clinics. In addition, information regarding various training formats could be gleaned, to assist others in similar training or the production of assessment tools. 
Since the prospect of experiencing some type of violence increases with the length of exposure to patients, nursing students' chances of being violated are increasing over time. All of the reviewed research agrees that nursing students need to learn verbal de-escalation techniques and other anti-aggression methods to protect themselves. The research has shown that de-escalation training can assist with therapeutic communication skills and knowledge globally, and the next section will outline its utilization in the United States.

\section{Methods}

In a perfect world, healthcare workers use the skills and knowledge for which they have been trained, able to feel safe knowing there will never be physical or verbal abuse. Unfortunately, this view does not reflect current reality (Beech \& Leather, 2003), nor has healthcare been without violence perhaps since its inception. Illness often brings out the worst in people, and occasionally violent and abusive behavior are the result (Beech, 1999).

The greatest risk of harm to any one group of healthcare workers is for student nurses (Beech \& Leather, 2003). Caught in a unique position between a triad of worldly inexperience, healthcare naiveté, and altruistically motivated behaviors, the student nurse may put him- or herself into the direct path of danger without even realizing the threat (Magnavita \& Heponiemi, 2011). Among the choices of tools which may prove beneficial to the student, especially in the early stages of the career, verbal de-escalation is this study's focus.

\subsection{Project Design}

This research project employed an experimental design which aimed to measure nursing student self-efficacy in verbal de-escalation skills (dependent variable), after receiving verbal de-escalation education (independent variable). Self-efficacy measurement was planned at pre-education and immediately post-education. Comparatively analyzing the differences in scores both before and after educational interventions assisted in determining the effect of such an intervention for nursing students during their psychiatric rotation. Since the purpose of the study pertained to whether there had been a numerical change in the level of self-efficacy, a quantitative study was an appropriate approach.

\subsection{Educational Intervention}

The educational intervention for the participants was an interactive presentation utilizing group exercises as well as facilitated discussion of brief case studies. Role-playing adapted for use with the nursing student population and prepared by the study author was a key learning activity. Various evidence-based guidelines and other articles of a similar nature provided the basic structure for the module, such as the Ten Domains recommended by the American Academy of Emergency Psychiatry (Richmond, Berlin, Fishkind, Holloman, Zeller, Wilson, \& $\mathrm{Ng}$, 2012). Included in the domains are respecting personal space, refraining from being provocative, and listening closely to what the patient is saying (Richmond et al., 2012, p. 20).

Each of these steps conveyed respect and a genuine caring while working to keep the situation and scenario unit safe. Questions and fears of the nursing students were addressed, as well as suggestions for communications with those who suffer mental and physical illness. Though this educational intervention was primarily directed toward nursing students who may come into contact with aggressive or agitated patients, there is also a growing concern in the country over lateral or peer violence (Beech and Leather, 2003; Tee et al., 2016). A portion of the presentation addressed the nursing profession's propensity to eat its young, and tips for coping with this type of anger.

\subsection{Sample and Setting}

The sample size of any study helps to determine the power of the conclusion and therefore can be a predictor of the width of the confidence interval (Corty \& Corty, 2011). A narrow confidence interval (CI) can be a greater predictor of the case for rejecting the null hypothesis. Planning for the optimal CI for the proposed research began with identifying the required sample.

\subsubsection{Sample}

The planned participants for the research project consisted of a convenience sample of ADN and BSN students from five different schools of nursing in the region. As a larger regional teaching hospital, the facility employing the author hosts three nursing schools which produce BSN students, and two that graduate ADN students. One of the BSN schools was a women's college, while the others registered both male and female students. All the schools were within a thirty- mile radius, while four were within five miles.

The mental health clinical rotation was in the second year of ADN programs and the third year for BSN programs. The BSN schools enrolled up to 20 students per semester, spread over two clinical groups of ten students each, for a potential sample frame of 60 students per regular 14-week semester. The ADN schools enroll 
up to ten students per half-semester, with a potential of 40 students per 14-week period. Therefore, the accessible population of mixed ADN and BSN students was a maximum of 100 each spring and fall 14-week term.

Exclusion criteria for this study sample included nursing students enrolled in a BSN completion, licensed practical nurse bridge program, or similar course of study due to the likelihood of previous professional work experience in a post-licensure environment. Beech (2008) outlined a similar concern for participants who rated themselves more self-efficacious than their counterparts in dealing with aggressive patients due to a more extensive patient interaction history and higher levels of self-respect after time in a professional role.

Other participants excluded were those unwilling or unable to complete the two parts of the survey process, administered before the intervention and immediately afterward, and four weeks following the intervention. Nursing students who were unable to complete the entire intervention of verbal de-escalation training, or in any other way did not meet inclusion criteria, were also-unable to participate in the research.

Nursing students in their mental health clinical rotation, male and female, any age, and possibly with the mental health clinical rotation out of sequence were included. The recruitment process was to be assisted by nursing instructors for each program by forwarding invitations to students for participation in the study. The instructors were also to provide specific information regarding length, frequency, and location of clinical rotation days.

Simulation activities have increased in popularity in the last several years as an adjunct or replacement for psychiatric inpatient experiences (Rose, Courey, Ball, Bowler, \& Thompson, 2012). Without significant live mental health patient experience after the verbal de-escalation educational intervention, there may be difficulty in the participants' ability to gauge self-efficacy with an aggressive patient. This difficulty was a weakness of the sampling strategy, as nursing schools may change the amount of student-to-patient time every semester, and indeed for every class.

The amount of time on the psychiatric units for each school may be widely variable and impacted by scheduling obstacles such as holidays. Though the sample size may be much less than anticipated, inferences to a similar target population were possible (Kline, 2016). The lack of generalizability was a potential weakness of this sampling method. One hundred participants was a small sample, resulting in a weakness of the study in the impact this sample size may have had upon the effect size, and therefore the power of the statistical test (Kline, 2016).

Even a priori calculations utilizing the G*Power 3.1 program (Faul, Erdfelder, Buchner, \& Lang, 2009) to elicit minimum sample sizes may have been inaccurate, (Kline, 2016). A Paired Samples T-Test with a medium effect size of 0.30 and a 0.05 level of significance yields an estimated power size of 0.95 , with a total sample size of 147 participants required. Lessening the power to 0.85 and keeping the level of significance at 0.05 and the effect size at 0.03 , the required sample size drops to 102 , but it is not known how these figures will be affected in the outcome (Kline, 2016).

A strength of the sampling strategy was the congruency in characteristics between the accessible population and the target population. If all nursing students were included and participated in the research, $60 \%$ would be from bachelor-degree schools. This figure is congruent with the current mix of bachelor- to associate-educated nurses employed on the psychiatric units of the clinical rotation facility at this time (P. Morrison, personal communication, January 14, 2018). Andrews (2014) estimated a ratio of a somewhat lesser value in 2014, at $41.5 \%$ of bachelor-educated direct-care nurses in the United States.

\subsubsection{Setting}

Originally, the 66-bed psychiatric facility of the researcher's employment was cited for the location of the proposed study. Embodying three adult units and one adolescent unit, all were housed in a location separate from the main medical hospital (though not considered freestanding for accreditation purposes). The adolescent unit admitted children aged thirteen to eighteen if still in school.

The three adult units were divided into an acute unit (akin to a psychiatric intensive care unit), a depression and subacute unit, and an older adult unit. The main units utilized by the nursing schools were the adult acute and depression units. The adult acute unit historically recorded the highest risk for violence, as most patients with such a risk or a high level of instability in behaviors were admitted there. Those adults with a lower risk of violence and more stable conditions such as schizoaffective and bipolar disorders were admitted to the depression and subacute units.

Support for the project was received from the outgoing and incoming Chief Nursing Officer and Vice President of Nursing, however in conference with the researcher's professors, there was a decision to perform the research at 
the schools of nursing, to avoid any appearance of conflict. There was no cost to the facility for the study. Costs of materials and supplies for the questionnaires were borne by the author.

\subsection{Instrumentation}

The tool utilized for this project was Thackrey's (1987) Clinical Confidence in Coping with Patient Aggression Instrument. Thackrey's instrument is a simple survey tool intended to measure the comfort level of the provider in handling patient aggression. The choice for this tool rested primarily on the two leading factors of appropriate fit for the proposed study, and construction of the tool itself. Thackrey's tool overtly introduced elements of a sample control group and follow-up during the instruments' testing phase, which other tools habitually eliminated with this type of research. The desire to be as rigorous as possible in this area of research meshed well with the instrument.

Developed while studying at Vanderbilt University, Thackrey (1987) used the tool to gauge the awareness of increasingly volatile patient disruptions in the mental health environment. Training programs to calm the agitated patient have been promoted widely, but a gold standard evaluation related to retention of information or the effectiveness of retained information and knowledge of appropriate action in a crisis event were nonexistent. The purpose of Thackrey's Instrument was to measure the self-confidence in using principles taught in an educational program of choice, by administering a survey before training and immediately afterward, and later (18 months in the original research).

Thackrey's tool (1987) consists of ten questions on a one through eleven Likert-type scale. The questions deal with comfort level in dealing with aggression (How comfortable are you in working with an aggressive patient?) through level of current training (How good is your present level of training for handling psychological aggression?) and abilities (How able are you to protect yourself physically from an aggressive patient?) to handle adverse events (Thackrey, 1987).

In testing construct validity, the initial version of the instrument tested fourteen questions on 53 clinicians, including therapists, nurses, and non-licensed assistants, of different educational levels, both male and female, all employed in a Veteran's Administration inpatient psychiatric unit and a state psychiatric prison (Thackrey, 1987). A second, larger study was conducted on staff of two outpatient community mental health centers, the prison, and a state psychiatric hospital. The tool was found to have a Cronbach's alpha coefficient of 0.92 , which is a high rating of interconnectedness of the items, and an appropriate test of a unidimensional survey (Thackrey, 1987). The possible range of scores on the bipolar ends of extreme low to extreme high is 10-110, with $S E=1.5$. A confidence level may be calculated from this information, so that an appropriate sample size may be figured. The instrument was said to have a high degree of internal consistency and stability, both measures are exhibits of reliability.

Alternatively, the Self-Efficacy Survey (SES) was considered for the measurement of self-efficacy during this research study (Panc, Mihalcea, \& Panc, 2012). Both the SES and Thackrey's tool are based on the theoretical stylings of Albert Bandura (1994). The primary tenet of the SES is that self-efficacy is the belief of one's ability to successfully behave in a particular manner and achieve one's objectives. Panc, Mihalcea, and Panc (2012) discussed Bandura's belief that persons with low self-esteem are less successful in carrying out desired actions, and that these failures cause setbacks in the self-esteem which eventually lead to illness such as depression.

One-hundred and four questions make up the Self-Efficacy Scale, scored on a one to six Likert scale, where one is equivalent to Strongly Disagree, and six is equivalent to Strongly Agree (Panc, Mihalcea, and Panc, 2012). In the early stages, the scale was whittled down from fifteen questions in each of the ten areas of intellectual, family, educational, professional, social, religious, erotic, moral, life standard, and health (150 questions in total) to only those that were relevant and acceptable to the two content experts.

Decreasing the questions by two to four items per area, the second sample pool's characteristics were matched as closely as possible (Panc, Mihalcea, and Panc, 2012), With the final version eliciting an internal consistency of 0.75 to 0.84 , the second version was superior to the wide-ranging 0.48 to 0.81 of the original version. Each of the ten areas related to various other scales which reinforced the adequacy of the Self-Efficacy Scales psychometric properties. The greater the score in each area and overall, the greater the level of self-efficacy around the ten areas. The authors of the scale reported the use of this tool for assessment of problem areas in psychiatric patients.

In utilization of the SES tool, the authors hoped to uncover the subject's deficiencies in multiple realms (Panc, Mihalcea, \& Panc, 2012). This is the point where the tool became clearly ill-suited for the purpose of gauging overall self-efficacy in the ability to communicate with an agitated or aggressive patient. The goal of this 
research project was not to discover the root cause of various aspects of the participants' character idiosyncrasies, but to focus on one specific area, regardless of perceived self-esteem or competencies in other areas. Additionally, nursing students are taught to distance their emotions and cover flaws from the patient bedside, making possible those tasks which would be otherwise hindered by the weight of feelings and stress (Birks, McKendree, \& Watt, 2009), therefore making the SES inappropriate for this project.

\subsection{Data Collection Method}

The execution of the research was realized in phases, maintaining transparency and academic rigor in each step (Ehrenstein, Christiansen, Schmidt, \& Sorensen, 2014). The first of these phases was the recruitment and consent process. The possible participant pool was to have been comprised of the mental health clinical rotation students from each of the five schools of nursing who utilized the facility for the clinical setting of psychosocial or mental health nursing.

In the three to four weeks prior to the research, communication with the schools of nursing provided data regarding final course enrollment figures, the exact timing and date of survey questionnaire distributions, and room reservation, if necessary. Institutional Review Board (IRB) approval was received from the university and each school of nursing. Preparation work had been accomplished via the deans of both types of programs (research and participant), development of the survey questionnaire, including the seven demographic questions, and the statistical codebook.

The clinical site's deans were also presented with detailed information on the study and permission granted from the IRB. Though research involving students may be exempt from requiring IRB approval from the nursing schools, two had expressed a need to make a cursory decision, nonetheless. After all approvals were obtained, information on the research specifics and an invitation to participate was to be distributed to prospective students via administrative assistants for each school and the email system, with posted flyers two weeks prior to the start of the semester.

At the beginning of the semester, the research study parameters were discussed thoroughly. The paper consent form was presented, reviewed line-by-line, and questions answered. After all concerns had been voiced, informed consent was requested. The students willing to participate in the research completed the pre-intervention survey, including demographic questions and writing their four- digit self-selected number plus first two letters of the mother's maiden name on the form. Students not wishing to participate in the research were nonetheless provided with verbal de-escalation education and kept housed in the same room as enrolled participants.

\subsection{Data Analysis Methods}

Once the forms were collected, a spreadsheet was prepared with the information contained on the surveys. The spreadsheet was password protected, and downloaded to a portable flash drive, then deleted from the desktop computer to avoid any unintentional sharing of data. The research codebook was also loaded into the spreadsheet for ease of calculation and reference.

The statistical codebook contained the coding as related to the variables of interest in the study and contained the original and recoded data once collected. The coding levels were aligned with the type of answer to each variable (Kim \& Mallory, 2017). Since all of Thackrey's (1987) questions relied on the use of a one to eleven Likert scale, those were coded as ordinal, without descriptors on the scale anchors. The original tool also did not utilize descriptors for every numeric entry on the Likert scale.

For the statistical analysis, the Paired Samples T-Test was utilized, for comparison of the means of a normally distributed dependent variable (such as the levels of self-efficacy) for two separate surveys of the same group (University of California Los Angeles: Institute for Digital Research and Education, 2018). The comparison of the means was meant to help answer the research question regarding a change in the level of self-efficacy with the learning module.

To test that the normal distribution of the means of scores, a Kolmogorov-Smirnov Test of Normality was performed (Pallant, 2016). If normality was not shown, a graphical representation in the form of a Q-Q Plot may have shed light on the number of data values which were out of alignment (Laerd Statistics, 2018). The Wilcoxan Signed- Rank Test was planned if the distribution of the means proved to be nonnormally distributed.

The testing procedures were to show the rejection or failure to reject the null hypothesis, which stated: The comparative scores between the pre-intervention and post-intervention surveys will show no difference on the Confidence in Coping with Patient Aggression scale. The null hypothesis would be rejected if the $p$-value was less than the level of significance, 0.05 . If the $p$-value was greater than the level of significance, the null 
hypothesis would not be rejected, or would have shown a failure to be rejected for the outcome of the study.

\subsection{Data Management Methods}

To minimize exposure of confidential student information, no information was collected for each student except for the demographic answers on the initial survey. After the informed consents were signed and collected, the students willing to participate in the research completed the pre-intervention survey, affixing the self-identified four-digit number and the first two letters of the mother's maiden name to minimize the chances of being able to identify the student or school. The surveys were collected into a large envelope after completion and were-sealed and put into a portable locked file box for transport back to the large locked file cabinet at the clinical site.

When all schools' surveys had been collected, the envelopes were unsealed, and data entered into an Excel spreadsheet. The spread sheet was password protected, downloaded onto a flash drive, and locked in a locking file cabinet with the survey forms. All research materials will remain in the locked filing cabinet for a period of five years. After the five years, the data forms will be destroyed via industrial shredding and the flash drive via hammer destruction, then incinerated.

\subsection{Ethical Considerations}

It is the responsibility of both the clinical instructor and the practicum site to ensure the student nurse has a safe learning experience (Anthony \& Wickman, 2015). Both the students and their patients are able to clearly validate the commitment to principles of safe care when faculty and nurses identify, evaluate, and inform the student of questionably safe behaviors. Therefore, providing information to increase the safety of the nursing student should be viewed as an ethical choice in which to engage prior to every psychiatric-mental health clinical rotation. Prior to the first clinical experience in mental health, the clinical instructor provided basic usual and customary safety information. This study was to originally include a control group, which would not have been given the educational intervention on verbal de-escalation, though the control group would receive the usual and customary information from the clinical instructor. Depriving the student of the verbal de-escalation education was not seen as an ethical concern by the primary investigator, as the pre-clinical education would be the normal standard information for all students. The study was of minimal risk to the participant nursing students. The probability and magnitude of harm or discomfort anticipated during this project was no different than that encountered in daily student life. Prior to the participation, the study was thoroughly explained, and all student questions were answered. The students could have chosen to decline participation in the research, without an adverse effect on their grade. The student did not participate in the research unless they fully understood the study, had all their questions answered, and were willing to voluntarily sign an informed consent (National Institutes of Health Clinical Center Department of Bioethics, n.d.). Despite these factors, the study design was forced into changing to a non-control scheme in order to pass the requirements of the investigator's university institutional review board.

\subsection{Internal and External Validity}

One potential threat to internal validity was testing sensitization (survey fatigue) due to the survey questions being repeated. This type of effect may be noted with behaviors such as straight-line answering, in which the participant answers all questions in a straight line down the column, and prematurely stopping the survey before completely answering all the questions. The short length of the surveys and lack of open-ended questions assisted in diminishing this possible side effect (O'Reilly-Shah, 2017).

Diffusion of verbal de-escalation educational module information was a distinct possibility, and an internal threat to the research. The students could have exchanged information using several electronic or low-fidelity platforms. During this type of activity, the group would behave as if receipt of the verbal de-escalation training had already occurred.

An external threat of lessened generalizability did exist in this study, in that the results may not be completely comparable to a similar study performed in another country, or in the United States, but using different content in the educational module. The level of usefulness will impact the scoring of self-efficacy because if the participants do not believe the information was useful, they are less likely to utilize it (Bandura, 1997). One way to decrease this threat is to ensure the inclusion of key elements, such as body language and communication style awareness.

\subsection{Summary}

Bandura (1977) theorized that the greater the level of self-efficacy, the greater the amount of behavioral functioning, such as that required for verbal de-escalation. This comparative prospective study used a randomized control design to investigate the self-efficacy levels of a convenience sample of nursing students. 
During the psychiatric-mental health clinical rotation, consenting students received up to 90 minutes of verbal de-escalation training. Thackrey's (1987) ten-question survey was administered before and immediately after education.

Data were analyzed using the Paired Samples T-Test to formulate an answer to the null hypothesis that there was no change in the level of self-efficacy between the two surveys. Human subject protection and informed consent was discussed, and confidentiality was maintained for all research participants and their data. The next section covers the results of the study, and discussion of the research findings.

\section{Results and Discussion of Findings}

The healthcare environment in the United States is one of the most dangerous places to work, with injury rates almost double that of private industry (Department of Labor, 2016). Registered nurses, and nursing students, are in the direct line of fire from agitated and aggressive patients (Jonas-Dwyer et al., 2017). Nursing students often lack the worldly experience and resources to care for these volatile patients, and often become targets from those with mental and substance abuse problems (Nau, Dassen, \& Halfens, 2009). The purpose of this section is to present the findings of the research, and to highlight the potential impact to the body of nursing knowledge for this vulnerable student population.

\subsection{Summary of Methods and Procedures}

A participant pool of 43 nursing students was surveyed on their self-efficacy in caring for patients who may be aggressive and potentially violent. The students were in their psychiatric-mental health course. Of the 43 possible participants, 26 were excluded due to previous work in the healthcare sector of over six months (not including nursing school cinical rotations).

A survey with seven demographic questions plus the ten questions of Thackrey's (1987) Confidence in Coping with Patient Aggression Instrument was completed prior to an educational intervention. The education was an interactive 60- to 90-minute session covering various aspects of verbal de-escalation, including practice scenarios of an agitated patient, a nurse, and frequently a nursing supervisor, all played by students. The researcher gave suggestions throughout the scenarios in language, attitude, communication style, and proxemics. The same survey instrument was given again, excluding the demographic questions. The two surveys were matched according to the students' self-selected identification code, and results tabulated.

The first set of scores of Thackrey's instrument were subtracted from the second, resulting in a positive change score. This change score was checked for normal distribution (Figure 1). The Kolmogorov-Smirnov Test of Normality showed a nonsignificant result $0.20(d f=17)$, meaning the scores were distributed fairly normally. A Paired Samples T-Test was conducted to evaluate the impact of the intervention on students' scores on the Clinican Confidence in Coping with Patient Aggression (CCCPA) scale. There was a statistically significant increase in CCCPA scores from pre-intervention $(M=39, S D=13.5)$ to post-intervention $(M=60.9, S D=$ $14.3), t(16)=6.92, p<.001$ (two-tailed). The mean increase in CCCPA scores was 21.8 with a $95 \%$ confidence interval ranging from 28.6 to 15.2 . The eta squared statistic (0.46) indicated a large effect size.

In the original research plan, three time periods were planned, representing survey completion before, immediately after, and four weeks following the verbal de-escalation education. However, a large portion of the student nurse sample was not available four weeks after the educational intervention, having moved onto other coursework. Institutional Review Board permission was granted for modification of the research to only the pre-and post-education surveys. Human subjects training standards were maintained. 


\subsection{Distribution of Score Differences}

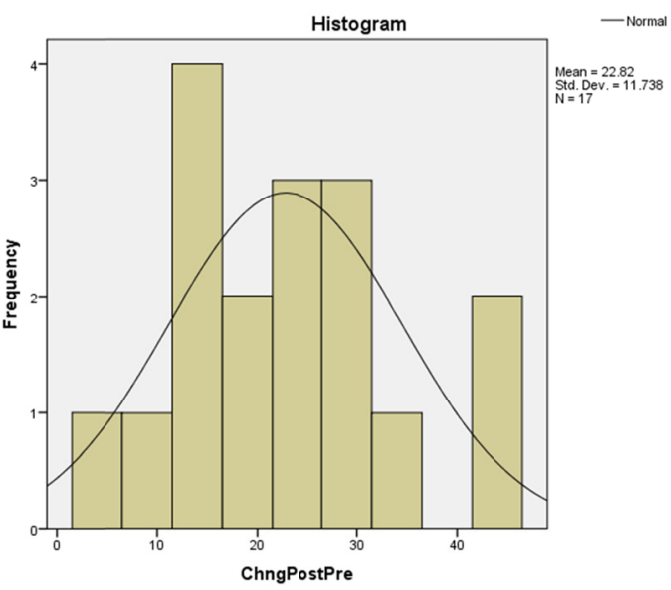

Figure 1. Distribution of the difference in scores between pre-intervention CCCPA scores and post-intervention CCCPA scores

\subsection{Summary of Sample and Setting Characteristics}

Research took place at three of the area's five schools of nursing. One of the original five schools was unable to approve the investigation for several months and so was excluded. Another school requested the research to be conducted outside of school hours; zero students stayed to participate. The three schools yielded 43 participants, $40 \%(n=17)$ from Bachelor programs of nursing, and $60 \%(n=26)$ from Associate programs (see Table 1), which was congruent with Andrews' (2014) estimates of the current nurse education mix. One of the strongest exclusion criterion was the presence of more than six months' work experience in healthcare, not including nursing school.

Those with greater healthcare work experience were hypothesized to have a higher chance of violence exposure and subsequent formation of coping skills to patient aggression and agitation. The survey tool questions assessed student self-efficacy in caring for such patients, which would likely increase with greater exposure (Bandura, 1977). Those with greater than six months' healthcare experience were therefore excluded. The resultant sample pool decreased to 17 female students. Of those 17 participants, eight were from Bachelor degree programs and nine were from Associate degree nursing programs.

Table 1. Total participant percentage of healthcare work experience (survey data in parentheses)

\begin{tabular}{llll}
\hline Characteristic & $\begin{array}{l}\text { Bachelor degree } \\
(n=17)\end{array}$ & $\begin{array}{l}\text { Associate degree } \\
(n=26)\end{array}$ & $\begin{array}{l}\text { Total } \\
(n=43)\end{array}$ \\
\hline Healthcare work $>6$ months & $53 \%(9)$ & $65 \%(17)$ & $60 \%(26)$ \\
\hline Healthcare work $<6$ months & $47 \%(8)$ & $35 \%(9)$ & $40 \%(17)$ \\
\hline Total & $100 \%(17)$ & $100 \%(26)$ & $100 \%(43)$ \\
\hline
\end{tabular}

The age of participants in the Bachelor programs ranged from less than 20 years $(n=6)$ to 20 to 30 years $(n=2)$. The age category of less than 20 years comprised $67 \%$ of the Bachelor program sample, while the larger portion in the Associate program $(n=5,71 \%)$ was in the older 20 to 30 year-old category (see Table 2$)$. Age was nonnormally distributed for the Bachelor programs, with skewness of $1.44(S E=0.75)$ and kurtosis of $0.000(S E$ $=1.48)$. Likewise for Associate programs, age was nonnormally distributed, with skewness of $0.25(S E=0.72)$ and kurtosis of $-0.040(S E=1.40)$. 
Table 2. Demographic characteristic percentages, by degree type (survey data in parentheses)

\begin{tabular}{|c|c|c|c|}
\hline \multirow[t]{2}{*}{ Characteristic } & Bachelor degree & Associate degree & Column Total \\
\hline & $47.1 \%(n=8)$ & $52.9 \%(n=9)$ & $100 \%(n=17)$ \\
\hline \multicolumn{4}{|l|}{ Average age } \\
\hline Less than 20 years & $67 \%(6)$ & $33 \%(3)$ & $52.9 \%(9)$ \\
\hline $20-30$ years & $29 \%(2)$ & $71 \%(5)$ & $41.2 \%(7)$ \\
\hline $31-40$ years & & $5.9 \%(1)$ & $5.9 \%(1)$ \\
\hline \multicolumn{4}{|l|}{ Gender } \\
\hline \multicolumn{4}{|l|}{ Male } \\
\hline Female & $47.1 \%(8)$ & $52.9 \%(9)$ & $100 \%(17)$ \\
\hline \multicolumn{4}{|l|}{ Semester in school } \\
\hline \multicolumn{4}{|l|}{$1^{\text {st }}$ semester } \\
\hline $2^{\text {nd }}$ semester & $31 \%(4)$ & $69 \%(9)$ & $76.5 \%(13)$ \\
\hline \multicolumn{4}{|l|}{$3^{\text {rd }}$ semester } \\
\hline $4^{\text {th }}$ semester & $23.5 \%(4)$ & & $23.5 \%(4)$ \\
\hline \multicolumn{4}{|c|}{ Previous de-escalation training } \\
\hline Yes & $25 \%(2)$ & $11 \%(1)$ & $18 \%(3)$ \\
\hline No & $75 \%(6)$ & $89 \%(8)$ & $82 \%(14)$ \\
\hline \multicolumn{4}{|c|}{ History of verbal or physical abuse by a patient } \\
\hline Yes & $5.9 \%(1)$ & & $5.9 \%(1)$ \\
\hline No & $44 \%(7)$ & $56 \%(9)$ & $94.1 \%(16)$ \\
\hline
\end{tabular}

Finally, only one participant (5.9\%) of the 17 selected particpants claimed to have experienced verbal or physical abuse by a patient in their nursing school career. Of the 26 participants excluded from the study (having more than six months' work experience in healthcare), $73 \%(n=19)$ reported experiencing verbal or physical abuse by a patient. Of those 19 students, four were in a Baccalaureate and 15 were enrolled in Associate programs. The majority $(n=5,71 \%)$ of the seven excluded participants who denied experiencing violence also denied previous de-escalation training. The seven were of varied age groups (two from age 20 or less, three from 20-30 years, one from 31-40, and one from 41-50 years), and four were in Baccalaureate programs while three were in Associate degree schools.

\subsection{Major Findings}

The major question in this study was regarding the potential impact on self-efficacy by as little as 90 minutes of verbal de-escalation training. A Paired Samples T-Test revealed a statistically significant increase in CCCPA scores following participation in the verbal de-escalation training program, suggesting a mental boost in the ability to handle aggressive patients, according to Bandura (1977). Echoing Nau, Dassen, Needham, and Halfen's 2009 research, Bandura's Self-Efficacy Theory (1977) was utilized as a structural cornerstone for the educational intervention.

While previous research recommended several hours to four days of verbal de-escalation training (Beech, 1999; Beech \& Leather, 2003; Brann \& Hartley, 2017; Gurkan \& Komurcu, 2017; Jonas-Dwyer et al., 2017; Magnavita \& Heponiemi, 2011; Needham et al., 2005), Bandura's Theory assisted in using performance accomplishment, vicarious experience, verbal persuasion, and emotional arousal throughout the 90-minute training to obtain personal mastery of the cognitive skills required for successful de-escalation. This finding expands on previous research findings for length of training, and for content.

Each of Self-Efficacy Theory's tenets worked well in practice to assist in engraining the verbal de-escalation principles. This basic program, interjected with personal stories, was interactive and individually meaningful enough to the nursing students for them to grasp the concepts needed, in a compressed format. The finding that significant results may be obtained in a shortened format adds to the base of knowledge of what is currently known about verbal de-escalation training and is congruent in length and objectives to Fernandes' shorter education intervention of four hours. 
The findings from this study also reiterate knowledge gained from previous studies in the age of nursing students and the prevalence of patient-generated verbal and physical aggression. Age of the selected nursing students in this study was most often less than 30 years, congruent with previous research (Jarvis \& Bhodraj, 2017; Nau, Dassen, Needham, Halfens, 2009; Sauer, Hannon, \& Beyer, 2017). Patient aggression toward nursing students has been reported at 25\% to 58\% (Hinchberger, 2009; Hopkins, Fetherston, \& Morrison, 2014; Jarvis \& Bhodraj, 2017; Nau, Dassen, Halfens, \& Needham, 2007; Needham et al., 2005; Wondrak \& Dolan, 1992). The rate of all 43 nursing students experiencing violence in this study was $47 \%(n=20)$.

\subsection{Implications for Nursing Practice}

The gaps in current research lie in the lack of public knowledge about the issue of healthcare violence, the lack of accountability on both the nursing school (education) and clinical site (practice) sides, and in a lack of faculty transparency about the prevalence and seriousness of the violence to nursing students and staff. Nursing students experience verbal and physical aggression from patients with increasing frequency in nursing clinical practice (Brann \& Hartley, 2017), including in this study. Such experiences have a ripple effect, as up to $60 \%$ of nurses consider leaving their position or profession within six months of graduating from school (Hinchberger, 2009).

In the midst of a nursing shortage (United States Department of Health and Human Services, 2002) and when there are less students being accepted into nursing schools (American Association of Colleges of Nursing, 2018), students need more reasons to consider nursing, not fewer. Nursing school faculty need to embrace the profession, including the problem areas, in a spirit of transparency. It is time to acknowledge each party's responsibility for the reality shock which occurs with the first nursing job- both the hospitals' and nursing schools' parts. A better-prepared new nurse is one which faculty have intentionally trained to weather the daily storms of patient care and comes to the practice environment with a sense of built-in resilience.

Nursing students and new graduates must be allowed to grow and learn in a nonthreatening environment so that they, too, do not promote the continuation of violence apathy. As soon as students begin to use the tools they have learned, they begin to change the patient-nurse relationship so that violence against nurses can begin to decrease, changing the culture in United States healthcare. A change in the healthcare environment which is based on intolerance for violence must be present throughout the healthcare culture, lest it creep back in when the focus is placed elsewhere.

\section{Recommendations}

Caring for numerous aggressive or agitated patients adds to the daily stress of a nursing student and may lead to disengagement (Martinez, 2017; Tee et al., 2016). When the staff fail to report incidents (Martinez, 2016) and inadvertently allow the violence to continue, the belief that nursing the sick and infirm means suffering abuse is internalized as part of the job (Brann \& Hartley, 2017). This research finds several areas in need of improvement in the nursing student's experience, including within the theory and practicum objectives. While the hospital culture is changing, the nursing school culture must simultaneously make changes. It is time for nursing education faculty in the United States to fully embrace the current state of workplace violence through education on its prevalence, and by starting the difficult conversations with students. Discussions focused on the realities and consequences of aggressive patients and healthcare violence provide an opportunity to correct misconceptions while discussing the problem in an open format, without fear of scaring the student away. Nursing faculty, students, and nurses in clinical practice must all be supported by nursing administration and assisted to change the culture through education for staff and patients, violence perpetrator prosecutions, increased compensation for higher-risk clinical practice areas, increased security and risk mitigation measures, and stronger local, regional, and national laws with explicit language warning of the consequences of violence to nurses, nursing students, and all healthcare workers.

One of the key concepts of Bandura's Theory of Self-Efficacy (1977) is that practice with a subject increases self-efficacy when dealing with the same topic later, leading to a greater chance to act correctly in a given (or emergent) situation. Unlike other investigations (Beech \& Leather, 2003; Nau et al., 2009; Zeller et al., 2006), this study verifies that effective verbal de-escalation training by nursing school faculty and clinical staff need not take an extensive amount of time to teach and can be accomplished in a two-hour block. Statistically and clinically significant results may be obtained from using a simplified program of an initial emotionally-arousing video on healthcare workplace violence, followed by PowerPoint slides and discussion on foundational topics including prevalence and legal aspects, an interactive personal boundary exercise and scenario role-playing in front of peers, and ending with a question and answer session.

Agitated-patient scenarios should be worked into multiple course simulations (Martinez, 2017) and case study practice just as medical complications are instilled. In this way, scenarios with a medical basis will be more 
realistic, and true to current patient populations. Faculty or clinical nurse-led simulation scenarios and debriefing assist in acknowledging the extent of the problem as well as-specific tools and communication suggestions to help combat violence. Simulation software companies such as Shadow Health (2018) have begun to incorporate mental health scenarios into their virtual reality platforms. More robust psychiatric issues need to also be incorporated into high-fidelity simulation manikins, realistically capturing the essence of the healthcare environment.

This research study adds to the limited research conducted with nursing students on the topic of healthcare violence in the United States. A larger study is recommended with the addition of a control to add rigor. The importance of demonstrating a dimension of self-efficacy in nursing students within their clinical environment, without the benefit of verbal de-escalation education, is required to gain a complete picture of healthcare violence and aggression. More in-depth demographic questions should be added, to probe into the specific details of workplace violence, including number, severity, and details of the incidents. The age groupings should be broken down into smaller categories to further pinpoint the prime age of risk for violence. Finally, further questions regarding the type of verbal-de-escalation training received would have been helpful, such as certification in a national program (such as Crisis Prevention Institute, 2009), through military service, or another option.

The last recommendation, missing from most previous research, is an outline of the specific education provided to the students. There has been debate over the content in the United Kingdom, specifically whether physical defense techniques should be taught. There would also be benefit in researching the verbal de-escalation education effect with nursing fundamentals education and post-education testing from four weeks to later in the nursing program, to see if the learned information was retained (Beech, 2003).

The research available on workplace violence with nursing students is quite limited, and further studies are encouraged due to the paucity of research, especially in the United States. Current and former graduate students should be encouraged to consider publication of studies regardless of the timeframe of the research, for the benefit of all, and those conducted in the area of workplace violence should be a priority. Education on the vitality of rigorous, high-standard nursing research should be mandatory, encouraged at all phases of nursing school, and more thoroughly supported in all venues in order to progress the knowledge of nursing care.

\section{Discussion}

This study builds on previous research which resulted in positive responses to verbal de-escalation training (Brann \& Hartley, 2017; Heckemann et al., 2015; Needham et al., 2005), and so may be applicable to nursing student populations outside of mental health. The project was similar to one conducted by Brann and Hartley (2017), with a participant pool of 40, which yielded statistically significant results. That study's authors utilized a homemade survey to measure mean scores pre- and post-intervention in workplace violence knowledge and awareness. Though the sample size was smaller $(n=17)$ in this research, the results were also statistically significant (while using a proven data collection instrument), but perhaps more importantly, were also clinically significant.

If the nursing students remembered only a part of their training and one less life-altering injury was accomplished as a result of verbal de-escalation education, the effort would have been worthwhile. Much of the previous research, including Beach and Leather (2003) and Nau (2010) taught similar content in the educational intervention as this research, including risk factors, incidence of violence occurrence, and safer approaches to patients. In contrast to Beech and Leather (2003) however, this study's intervention did not include teaching physical restraint or takedown methods. This author is in agreement with Beech (1999) that there is an inherent responsibility in teaching physical intervention techniques to practice, retrain, coaching through situations, and debriefing afterward, thereby making these techniques outside the scope of this project.

The length of the shortest educational intervention in previous research was four hours in total (Fernandes et al., 2002). In contrast, this project's educational intervention lasted just 90 minutes, a relatively short amount of time that could be worked into most curriculum schedules. Though all of the previous research was completed with no intention to repeat the training, the Crisis Prevention Institute (2009) recommends training in verbal de-escalation techniques at least annually. This educational intervention was intended to be conducted once, though the outline for the program was made available to each school. Within the healthcare industry, companies such as the one wherein this author is employed, now realize the benefit of aggressive patient scenario drills intermittently to keep skills fresh.

The strengths of the reviewed literature for this study align with the strengths of this research project: the interest of nursing students in learning about workplace violence, and defining choices when faced with an agitated 
patient (Beech \& Leather, 2003; Brann \& Hartley, 2017; Grenyer et al., 2004). The commitment of researchers and faculty to improving the quality of learning for students through measures that help to ensure their safety at clinical rotation sites was universal (Heckemann et al., 2015), and few of the nursing students declined to participate in this study. Several students expressed gratitude after the training. Teaching verbal de-escalation in the nursing student classroom is a timely mission, as healthcare remains exponentially more violent than other industries year after year (United States Department of Labor, 2016).

One of the weaknesses of this research study was the small sample size ( $n=17$ after exclusions). The participant pool meeting all study requirements was not anticipated to be cut by nearly two-thirds, indicating that many nursing students may have been either working in healthcare while simultaneoulsy attending school, or began as nurse extenders before deciding to further their career. Participant pool populations could be increased by either extending the school location boundaries out to 75 to 100 miles or building on successive nursing cohorts within the same geographic location, thereby extending the timeline of the study.

Another weakness of the study was the lack of research conducted in the United States on this topic, with student nurses. Dissertations and other research-based projects within the United States which focused on nursing students and workplace violence were not easy to find; Brann and Hartley's 2017 study utilizing a free computer-based intervention was the only article available. More information would have been beneficial to the current study in the navigation of collegiate, healthcare, and governmental structures, as well as to more accurately contextualize findings of the study to populations in the current nursing school environment. Additionally, such research from this country would exhibit an ability to compete in the global marketplace (Jeffrey, 2006).

Though the main goal for this project was focused on nursing students and aggressive patients, information was presented in the educational intervention on lateral violence as well. The lack of focus on bullying and lateral violence in data collection was yet another weakness of this research project. Ostensibly, violence is violence, and any assault may have traumatic effects regardless of the perpetrator. Greater efforts to delineate lateral nurse on nurse violence, including bullying, could occur in future research. The decades-old behaviors and attitudes of nursing eating its young needs to be dissected, and its component parts brought to light and dealt with definitively.

The original study design was changed significantly to meet Institutional Review Board requirements, though was still able to be completed sufficiently. Large world-renowned research institutions, such as Johns Hopkins University, should consider formulating research guidelines, similar to clinical standards of care, for national adoption to assimilate all students to a minimum level of scholarship. Areas such as psychiatric nursing and the best-practice care of patients with mental health issues have been underdeveloped, leading to few changes in the care of this patient population over the last ten years. High-quality research is needed in greater quantity in order to assist in changing the care and culture of today's psychiatric inpatient units.

\section{Conclusions and Contributions to the Profession of Nursing}

Nursing is one of the most high-risk professions for violence (Department of Labor, 2016), yet many consider the caring professions an innate part of their characterological makeup. Nursing students likewise are at risk, and perhaps no less passionate, experiencing violence in numbers equivalent to the 25 to $58 \%$ noted in licensed nurses (Hinchberger, 2009; Hopkins, Fetherston, \& Morrison, 2014; Jarvis \& Bhodraj, 2017; Nau, Dassen, Halfens, \& Needham, 2007; Needham et al., 2005); 47\% in this study group.

Verbal de-escalation is an evidence-based practice for the care of agitated patients (Martinez, 2016). Both clinical sites and nursing education have a responsibility to optimize the nursing student's experience (Beech, 1999; Benner, Sutphen, Leonard, \& Day, 2010; Shinn, 2001). Verbal de-escalation education has shown to assist in helping the student feel more efficacious in previous studies (Heckemann, 2015), and in this one. This research has reiterated the effectiveness of specific teaching strategies and content to promote student efficacy (Nau et al., 2009).

Workplace violence occurs in all countries, cultures, and venues in the modern world (Hopkins, Fetherston, \& Morrison, 2014). When persons with harmful intent interrupt healthcare work, others are harmed, directly and indirectly (Campbell, 2011). Nursing students, by definition, practice their budding skills on potentially harmful persons without full knowledge, awareness, or preparation in dealing with aggression (Nau et al., 2007; Nau et al., 2009; Needham et al., 2005). This project has contributed to the improvement of nursing practice by equipping student nurses and faculty with the knowledge needed to add verbal de-escalation skills to each future nurses' clinical skill toolbox. 
Additionally, nursing schools may feel more comfortable with using live patients for their clinical experience instead of replacing this time with simulation. The students' knowledge of violence assessment and prevention techniques helps to decrease the risk for their involvement in adverse circumstances. Results of studies such as in Beech (2007) may be utilized in training to specifically highlight that nursing students on a clinical rotation should not think of themselves as junior nurses with more experience and know-how than mental health technicians working on the unit. When simulation is part of the curriculum, agitated patients need to be included in scenarios to practice and reinforce verbal de-escalation skills (Martinez, 2017).

Efforts to train current hospital and healthcare staff requires continued infiltration down every hallway and in every business and medical office. Nursing students experience workplace violence in numbers rivaling registered nurses (Hinchberger, 2009) from patients and peers (bullying), but also in nursing school (Magnavita \& Heponiemi, 2011). Anti-violence training needs to be an internal attitude, value, and culture change (Heckemann et al., 2015), focused on competencies which exhibit detection and removal of aggression causes, correct assessment of agitation situations, verbal de-escalation skill, and coping with personal stress (Nau et al., 2007). A consistent approach within nursing school and healthcare environments sends a message of the violence problem acknowledgement, nurse and human life value, and respect for all persons, regardless of gender, race, profession, or ability.

\section{References}

Abuse. (2017). Merriam-Webster Dictionary online. Retrieved from https://www.merriam-webster.com/dictionary/abuse

American Association of Colleges of Nursing. (2018). AACN fact sheet. Retrieved from http://www.aacnnursing.org/News-Information/Fact-Sheets/AACN-Fact-Sheet

Andrews, N. (2014). Advancing toward an $80 \%$ BSN workforce by 2020. Retrieved from https://www.americannursetoday.com/advancing-toward-an-80-bsn-workforce-by-2020/

Anthony, M., \& Wickman, M. (2015). Precepting challenges. Nurse Educator, 40(3), 113-114. https://doi.org/10.1097/NNE0000000000000118

Bandura, A. (1977). Self-efficacy: Toward a unifying theory of behavioral change. Psychological Review, 84(2), 191-215. https://doi.org/10.1037/0033-295x.84.2.191

Bandura, A. (1993). Perceived self-efficacy in cognitive development and functioning. Educational Psychologist, 28(2), 117-148. https://doi.org/10.1207/s15326985ep2802_3

Bandura, A. (1994). In V. Ramachaudran (Ed.), Encyclopedia of human behavior (Vol. 4). New York: Academic Press.

Bandura, A. (1997). Self-efficacy: The exercise of control (1st ed.). New York, NY: Worth.

Beech, B. (1999). Sign of the times or the shape of things to come? A 3-day unit of instruction on 'aggression and violence in health settings for all students during pre-registration nurse training'. Nurse Education Today, 19, 610-616. https://doi.org/10.1054/nedt.1999.0356

Beech, B. (2008). Aggression prevention training for student nurses: Differential responses to training and the interaction between theory and practice. Nurse Education in Practice, 8(2), 94-102. https://doi.org/10.1016/j.nepr.2007.04.004

Beech, B., \& Leather, P. (2003). Evaluating a management of aggression unit for student nurses. Journal of Advanced Nursing, 44(6), 603-612. https://doi.org/10.1046/j.0309-2402.2003.02850.x

Benner, P., Sutphen, M., Leonard, V., \& Day, L. (2010). Educating nurses: A call for radical transformation. San Francisco, CA: Jossey-Bass Publishers.

Birks, Y., McKendree, J., \& Watt, I. (2009). Emotional intelligence and perceived stress in healthcare students: A multi-institutional, multi-professional survey. BMC Medical Education, 9(1), 61. https://doi.org/10.1186/1472-6920-9-61

Brann, M., \& Hartley, D. (2017). Nursing student evaluation of NIOSH workplace violence prevention for nurses' online course. Journal of Safety Research, 60, 85-91. https://doi.org/10.1016/j.jsr.2016.12.003

Campbell, J., Messing, J., Kub, J., Agnew, J., Fitzgerald, S., Fowler, B., ... Bolyard, R. (2011). Workplace violence: Prevalence and risk factors in the Safe at Work Study. Journal of Occupational and Environmental Medicine, 53(1), 82-89. https://doi.org/10.1097/JOM.0b013e3182028d55 
Centers for Disease Control and Prevention. (2014). Substance use and mental disorders: Early detection, prevention, and treatment [PowerPoint slides]. Retrieved from https://www.cdc.gov/nchs/ppt/hp2020/hp2020_mh_md_and_sa_progress_review_presentation.pdf

Centers for Disease Control and Prevention. (2015, May 24). Occupational Traumatic Injuries Among Workers in Health Care Facilities -- United States, 2012-2014, weekly. Retrieved from https://www.cdc.gov/mmwr/preview/mmwrhtml/mm6415a2.htm

Christiansen, C., Schmidt, M., \& Sorenson, H. (2014). Non-experimental comparative effectiveness research: How to plan and conduct a good study. Current Epidemiology Reports, 1(4), 206-212. https://doi.org/10.1007/s40471-014-0021-5

Cleary, M., Horsfall, J., \& DeCarlo, P. (2006). Improving student learning in mental health settings: The views of clinical stakeholders. Nurse Education in Practice, 6(3), 141-148. https://doi.org/10.1016/j.nepr.2005.11.003

Confidence. (2017). In Merriam-Webster Dictionary online. Retrieved from https://www.merriam-webster.com/dictionary/confidence

Corty, E., \& Corty, R. (2011). Setting sample size to ensure narrow confidence intervals for precise estimation of population values. Nursing Research, 60(2), 148-153. https://doi.org/10.1097/NNR.0b013e318209785a

Cowin, L., Davies, B., Estall, T., Fitzgerald, M., \& Hoot, S. (2003). De-escalating aggression and violence in the mental health setting. International Journal of Mental Health Nursing, 12, 64-73. https://doi.org/10.1046/j.1440-0979.2003.00270.x

Crisis Prevention Institute. (2009). Joint Commission standards on restraint and seclusion: Nonviolent Crisis Intervention training program. from https://www.crisisprevention.com/CPI/media/Media/Resources/alignments/Joint-Commission-Restraint-Sec lusion-Alignment-2011.pdf

Faul, F., Erdfelder, E., Buchner, A., \& Lang, A. (2009). Statistical power analyses using G*Power 3.1: Tests for correlation and regression analyses. Behavioral Research Methods, 41(4), 1149-1160. https://doi.org/10.3758/BRM.41.4.1149

Fernandes, C., Raboud, J., Christensen, J., Bouthillette, F., Bullock, L., Ouellet, L., \& Moore, C. (2002). The effect of an education program on violence in the emergency department. Annals of Emergency Medicine, 39(1), 47-55. https://doi.org/10.1067/mem.2002.121202

Fiedler, R., Breitenstein, S., Delaney, K. (2012). As assessment of students' confidence in performing psychiatric mental health nursing skills: The impact of the clinical practicum experience. Journal of the American Psychiatric Nurses Association, 18(4), 244-250.

Grenyer, B., Ilkiw-Lavalle, O., Biro, P., Middleby-Clements, J., Comninos, A., \& Coleman, M. (2004). Safer at work: Development and evaluation of an aggression and violence minimization program. Australian and New Zealand Journal of Psychiatry, 38, 804-810. https://doi.org/10.1080/j.1440-1614.2004.01465.x

Gurkan, O., \& Komurcu, N. (2017). The effect of a peer education program on combating violence against women: A randomized controlled study. Nurse Education Today, 57, 47-53. https://doi.org/10.1016.j.nedt.2017.07.003

Heckemann, B., Zeller, A., Hahn, S., Dassen, T., Schols, J., \& Halfens, R. (2015). The effect of aggression management training programmes for nursing staff and students working in an acute hospital setting: A narrative review of current literature. Nursing Education Today, 35(1), 212-219. https://doi.org/10.1016/j.nedt.2014.08.003

Hinchberger, P. (2009). Violence against female student nurses in the workplace. Nursing Forum, 44(1), 37-46. https://doi.org/10.1111/j.1744-6198.2009.00125.x

Hopkins, M., Fetherston, C., \& Morrison, P. (2014). Prevalence and characteristics of aggression and violence experienced by Western Australian nursing students during clinical practice [Supplemental material]. Contemporary Nurse, 49(1), 113-121.

Jarvis, M., \& Bhodraj, L. (2017). Student nurses' perceptions of district hospital resource adequacy for aggression management of mental health care users. African Journal of Nursing and Midwifery, 19(1), 118-130. https://doi.org/10.25159/2520-5293/837 
Jeffrey, W. (2006). Importance of basic research to United States' competitiveness. Retrieved from https://www.nist.gov/speech-testimony/importance-basic-research-united-states-competitiveness

Jonas-Dwyer, D., Gallagher, O., Saunders, R., Dugmore, H., Bulsara, C., \& Metcalfe, H. (2017). Confronting reality: A case study of a group of student nurses undertaking a management of aggression training (MOAT) program. Nurse Education in Practice, 27, 78-88. https://doi.org/10.1016/j.nepr.2017.08.008

Kim, M., \& Mallory, C. (2017). Statistics for evidence-based practice in nursing (2nd ed.). Burlington, MA: Jones \& Bartlett Learning.

Kline, T. (2016). Sample issues, methodological implications, and best practices. Canadian Journal of Behavioural Science, 49(2), 71-77. https://doi.org/10.1037/cbs0000054

Kraiger, K., Ford, K., \& Salas, E. (1993). Application of cognitive, skill-based, and affective theories of learning outcomes to new methods of training evaluation. Journal of Applied Psychology, 78(2), 311-328. https://doi.org/10.1037/0021-9010.78.2.311

Laerd Statistics. (2018). Independent t-test for two samples. Retrieved from https://statistics.laerd.com/statistical-guides/independent-t-test-statistical-guide.php

Lavell, M., Stewart, D., James, K., Richardson, M., Renwick, L., Brennan, G., \& Bowers, L. (2016). Predictors of effective de-escalation in acute inpatient psychiatric settings. Journal of Clinical Nursing, 25, 2180-2188. https://doi.org/10.1111/jocn.13239

Magnavita, N., \& Heponiemi, T. (2011). Workplace violence against nursing students and nurses: An Italian $\begin{array}{lllll}\text { experience. } \quad \text { Journal } & \text { of } & \text { Nursing } & \text { Scholarship, } & \text { 43(2), }\end{array}$ https://doi.org/10.111/j.1547-5069.2011.01392.x

Mahoney, J., Palyo, N., Napier, G., \& Giordano, J. (2009). The therapeutic milieu reconceptualized for the 21 stcentury. Archives of Psychiatric Nursing, 23(6), 423-429. https://doi.org/10.1016/j.apnu.2009.03.002

Martinez, A. (2016). Managing workplace violence with evidence-based interventions: A literature review. Journal of Psychosocial Nursing, 54(9), 31-36. https://doi.org/10.3928/02793695-20160817-05

Martinez, A. (2017). Implementing a workplace violence simulation for undergraduate nursing students: A pilot study [Abstract]. Journal of Psychosocial Nursing and Mental Health Services, 55(10), 39-44.

McGowan, S., Wynaden, D., Harding, N., Yassine, A., \& Parker, J. (1999). Staff confidence in dealing with aggressive patients: A benchmarking exercise. Australian and New Zealand Journal of Mental Health Nursing, 8, 104-108. https://doi.org/10.1046/j.1440-0979.1999.00140.x

National Institutes of Health Clinical Center Department of Bioethics. (n.d.). Research ethics: How to treat people who participate in research. https://bioethics.nih.gov/education/FNIH_BioethicsBrochure_WEB.PDF

Nau, J., Dassen, T., Halfens, R., \& Needham, I. (2007). Nursing students' experiences in managing patient aggression. Nurse Education Today, 27(8), 933-946. https://doi.org/10.1016/j.nedt.2007.01.007

Nau, J., Dassen, T., Needham, I., \& Halfens, R. (2009a). The development and testing of a training course in aggression for nursing students: A pre- and post-test study. Nurse Education Today, 29(2), 196-207. https://doi.org/10.1016/j.nedt.2008.08.011

Nau, J., Halfens, R., Needham, I., \& Dassen, T. (2010). Student nurses' de-escalation if patient aggression: A pretest-posttest intervention study. International Journal of Nursing Studies, 47(6), 699-708. https://doi.org/10.1016/j.ijnurstu.2009.11.011

Nau, J., Needham, I., Dassen, T., \& Halfens, R. (2009b). Development and psychometric testing of the De-escalating Aggressive Behavior Scale. Journal of Advanced Nursing, 65(9), 1956-1964. https://doi.org/10.1111/j.1365-2648.2009.05087.x

Needham, I., Abderhalden, C., Dassen, T., Haug, H., \& Fischer, J. (2004). The perception of aggression by nurses: Psychometric scale testing and derivation of a short instrument. Journal of Psychiatric and Mental Health Nursing, 11(1), 36-42. https://doi.org/10.1111/j.1365-2850.2004.00678.x

Needham, I., Abderhalden, C., Zeller, A., Dassen, T., Haug, H., Fischer, J., \& Halfens, R. (2005). The effect of a training course on nursing students' attitudes toward, perceptions of, and confidence in managing patient aggression. Journal of Nursing Education, 44(9), 415-420. 
O'Reilly-Shah, V. (2017, September 12). Factors influencing healthcare provider respondent fatigue answering a globally administered in-app survey [Supplemental material]. Peer J- the Journal of Life and Environmental Sciences. https://doi.org/10.7717/peerj.3785

Pallant, J. (Ed.). (2016). SPSS survival manual: A step by step guide to data analysis using IBM SPSS (6th ed.). New York: McGraw Hill Education.

Panc, T., Mihalcea, A., \& Panc, I. (2012). Self-Efficacy Survey: A new assessment tool. Procedia - Social and Behavioral Sciences, 33, 880-884. https://doi.org/10.1016/j.sbspro.2012.01.248

Quail, M., Bundage, S., Spitalnick, J., Allen, P., \& Beilby, J. (2016). Student self-reported communication skills, knowledge, and confidence across standardized patient, virtual and traditional clinical learning environments. BMC Medical Education, 16(73), 1-16. https://doi.org/10.1186/s12909-016-0577-5

Random.org. (2018). List randomizer. Retrieved from https://www.random.org/lists/

Richmond, J., Berlin, J., Fishkind, A., Holloman, G., Zeller, S., Wilson, M., ... Ng, A. (2012). Verbal de-escalation of the agitated patient: Consensus statement of the American Association for Emergency Psychiatry Project BETA de-escalation workgroup. Western Journal of Emergency Medicine, 13(1), 17-25. https://doi.org/10.5811/westjem.2011.9.6864

Rose, S., Courey, T., Ball, M., Bowler, C., \& Thompson, Z. (2012). Bringing simulation to life through a therapeutic encounter: A pedagogical approach used for associate degree nursing students. Teaching and Learning in Nursing, 7, 2-5. https://doi.org/10.1016/j.teln.2011.09.003

Sauer, P., Hannon, A., \& Beyer, K. (2017). Peer incivility among prelicensure nursing students. Nurse Educator, 42(6), 281-285. https://doi.org/10.1097/NNE.0000000000000375

Sherer, M., Maddux, J., Mercandante, B., Prentice-Dunn, S., Jacobs, B., \& Rogers, R. (1982). The Self-Efficacy Scale: Construction and validation. Psychological Reports, 51(2), 663-671. https://doi.org/10.2466/pr0.1982.51.2.663

Shinn, L. (2001). The nursing risk management series: Yes, you can be sued. Retrieved from http://ana.nursingworld.org/mods/archive/mod310/cerm101.htm

Simon, T., \& Hurvitz, K. (2014). Healthy People 2020 objectives for violence prevention and the role of nursing. The Online Journal of Issues in Nursing, 19(1). https://doi.org/10.3912/OJIN.Vol19No1Man01

Speroni, K., Fitch, T., Dawson, E., Dugan, L., \& Atherton, M. (2014). Incidence and cost of nurse workplace violence perpetrated by hospital patients or patient visitors. Journal of Emergency Nursing, 40(3), 218-228. https://doi.org/10.1016/j.jen.2013.05.014

Tee, S., Ozcetin, Y., \& Russell-Westhead, M. (2016). Workplace violence experienced by nursing students: A UK study. Nursing Education Today, 41, 30-35. https://doi.org/10.1016/j.nedt.2016.03.014

Thackrey, M. (1987). Clinician confidence in coping with patient aggression: Assessment and enhancement. Professional Psychology: Research and Practice, 18(1), 57-60.

Truman, A., Goldman, M., Lehna, C., Berger, J., \& Topp, R. (2013). Verbal abuse of pediatric nurses by patients and families. Kentucky Nurse, 61(1), 6-8.

United States Department of Health and Human Services. (2002, July). Projected supply, demand, and shortages of registered nurses: 2000-2020. Retrieved from https://www.ahcancal.org/research_data/staffing/Documents/Registered_Nurse_Supply_Demand.pdf

United States Department of Labor. (2016). Occupational Safety and Health Administration: Worker Safety in Hospitals: Caring for our caregivers. Retrieved from https://www.osha.gov/dsg/hospitals/

University of California Los Angeles: Institute for Digital Research and Education. (2018). What statistical analysis should I use? Statistical analyses using SPSS. Retrieved from https://stats.idre.ucla.edu/spss/whatstat/what-statistical-analysis-should-i-usestatistical-analyses-using-spss/ \#2ittest

Unsworth, J., McKeever, M., \& Kelleher, M. (2012). Recognition of physical deterioration in patients with mental health problems: The role of simulation in knowledge and skill development. Journal of Psychiatric and Mental Health Nursing, 19(6), 536-545. https://doi.org/10.1111/j.1365-2850.2011.01828.x

Whitley, G., Jacobsen, G., \& Gawrys, M. (1996). The impact of violence in the health care setting upon nursing education. Journal of Nursing Education, 35(5), 211-218. 
Wondrak, R., \& Dolan, B. (1992). Dealing with verbal abuse: Evaluation of the efficacy of a workshop for student nurses. Nurse Education Today, 12, 108-115. https://doi.org/10.1016/0260-6917(92)90036-N

World Health Organization. (2018). Violence and injury prevention. Retrieved from http://www.who.int/violence_injury_prevention/violence/activities/workplace/en/

\section{Copyrights}

Copyright for this article is retained by the author(s), with first publication rights granted to the journal.

This is an open-access article distributed under the terms and conditions of the Creative Commons Attribution license (http://creativecommons.org/licenses/by/4.0/). 